\title{
FIBERS OF PARTIAL TOTALIZATIONS OF A POINTED COSIMPLICIAL SPACE
}

\author{
AKHIL MATHEW AND VESNA STOJANOSKA \\ (Communicated by Michael A. Mandell)
}

\begin{abstract}
Let $X^{\bullet}$ be a cosimplicial object in a pointed $\infty$-category. We show that the fiber of $\operatorname{Tot}_{m}\left(X^{\bullet}\right) \rightarrow \operatorname{Tot}_{n}\left(X^{\bullet}\right)$ depends only on the pointed cosimplicial object $\Omega^{k} X^{\bullet}$ and is in particular a $k$-fold loop object, where $k=$ $2 n-m+2$. The approach is explicit obstruction theory with quasicategories. We also discuss generalizations to other types of homotopy limits and colimits.
\end{abstract}

\section{INTRODUCTION}

Let $X^{\bullet}$ be a pointed cosimplicial space, that is, $X^{\bullet}$ is a functor $\Delta \rightarrow \mathcal{S}_{*}$ from the simplex category $\Delta$ to the $\infty$-category $\mathcal{S}_{*}$ of pointed spaces. The totalization Tot $X^{\bullet}$ is defined to be the homotopy limit of $X^{\bullet}$.

To understand $\operatorname{Tot} X^{\bullet}$, it is convenient to filter the category $\Delta$. Let $\Delta \leq n$ be the subcategory of $\Delta$ spanned by the nonempty totally ordered sets of cardinality $\leq n+1$. Then $\operatorname{Tot} X^{\bullet}$ is the homotopy inverse limit of the tower

$$
\cdots \rightarrow \operatorname{Tot}_{n}\left(X^{\bullet}\right) \rightarrow \operatorname{Tot}_{n-1}\left(X^{\bullet}\right) \rightarrow \cdots \rightarrow \operatorname{Tot}_{1}\left(X^{\bullet}\right) \rightarrow \operatorname{Tot}_{0}\left(X^{\bullet}\right)=X^{0},
$$

where $\operatorname{Tot}_{n}\left(X^{\bullet}\right)$ is the homotopy limit of the diagram $\left.X^{\bullet}\right|_{\Delta \leq n}$.

The tower (11) is extremely useful. For example, it leads to a homotopy spectral sequence [4, Ch. X] for computing the homotopy groups of the totalization (at the specified basepoint). For simplicity, we assume all fundamental groups abelian and all $\pi_{1}$-actions on higher homotopy groups trivial. Then the $E_{2}$-term of this spectral sequence can be determined purely algebraically, if one knows the homotopy groups of $X^{\bullet}$. Namely, for each $t$, one obtains a cosimplicial abelian group $\pi_{t} X^{\bullet}$, and $E_{2}^{s, t}$ is the $s$ th cohomology group of the associated complex. This spectral sequence is a basic tool of algebraic topology.

It is classical that the fibers of the maps $\operatorname{Tot}_{m}\left(X^{\bullet}\right) \rightarrow \operatorname{Tot}_{m-1}\left(X^{\bullet}\right)$ are naturally $m$-fold loop spaces. To be specific, let $M^{m} X^{\bullet}$ be the $m$ th matching space of the cosimplicial space $X^{\bullet}$, that is,

$$
M^{m} X^{\bullet}=\lim _{\substack{[m+1] \rightarrow[k] \\ k \leq m}} X^{k} .
$$

We have a natural map $X^{m} \rightarrow M^{m-1} X^{\bullet}$ with fiber denoted $N^{m} X^{\bullet}$, and the fiber of $\operatorname{Tot}_{m}\left(X^{\bullet}\right) \rightarrow \operatorname{Tot}_{m-1}\left(X^{\bullet}\right)$ can be identified with the $m$-fold loop space of $N^{m} X^{\bullet}$.

Received by the editors August 12, 2014 and, in revised form, December 10, 2014 and December $18,2014$.

2010 Mathematics Subject Classification. Primary 55U35, 55U40.

The first author was partially supported by the NSF Graduate Research Fellowship under grant DGE-110640.

The second author was partially supported by NSF grant DMS- 1307390 . 
In particular, it depends only on $\Omega^{m} X^{\bullet}$, as $M^{m-1} X^{\bullet}$ is defined as a limit and therefore commutes with $\Omega^{m}$.

The main result of this note is that one can obtain a similar picture for the fibers of $\operatorname{Tot}_{m}\left(X^{\bullet}\right) \rightarrow \operatorname{Tot}_{n}\left(X^{\bullet}\right)$ for $n \leq m$, in a restricted range, and in any pointed $\infty$-category, as follows.

Theorem 1. Let $n \leq m \leq 2 n+1$ and let $\mathcal{C}$ be any pointed $\infty$-category with limits. There is a limit-preserving functor $F_{n, m}: \operatorname{Fun}(\Delta, \mathcal{C}) \rightarrow \mathcal{C}$ such that there exists, for any $X^{\bullet} \in \operatorname{Fun}(\Delta, \mathcal{C})$, a functorial fiber sequence in $\mathcal{C}$,

$$
F_{n, m}\left(\Omega^{r} X^{\bullet}\right) \simeq \Omega^{r} F_{n, m}\left(X^{\bullet}\right) \rightarrow \operatorname{Tot}_{m}\left(X^{\bullet}\right) \rightarrow \operatorname{Tot}_{n}\left(X^{\bullet}\right)
$$

where $r=2 n-m+2$.

In less formal terms, the fiber of $\operatorname{Tot}_{m}\left(X^{\bullet}\right) \rightarrow \operatorname{Tot}_{n}\left(X^{\bullet}\right)$ depends only on $\Omega^{r} X^{\bullet}$ as a cosimplicial object (and this fiber is in particular an $r$-fold loop space). Our arguments are based on elementary obstruction-theoretic techniques, but in the setting of the quasicategory model of $\infty$-categories. They in particular apply to certain instances of homotopy (co)limits over finite posets such as the poset of nontrivial subspaces of a finite-dimensional vector space.

However, as a result, we do not obtain an explicit description of the functor $F_{n, m}$ : its existence is established by showing that a certain combinatorially defined functor desuspends $r$ times. We show that such a desuspension exists by obstruction theory, but we do not write it down.

In a similar vein, we obtain the following result.

Theorem 2. Let $X$ be a pointed topological space covered by $n$ open sets $U_{1}, U_{2}, \ldots$, $U_{n} \subset X$, each containing the basepoint. Suppose the intersection of any collection of at most $r$ of the $\left\{U_{i}\right\}$ is weakly contractible. Then $X$ has the weak homotopy type of a $(2 r-n+1)$-fold suspension.

Results of a similar flavor in Goodwillie calculus have been obtained by AroneDwyer-Lesh [1].

\section{Deloopable subcategories}

We first consider this question more generally. Let $\mathcal{D}$ be an $\infty$-category, and let $\mathcal{C} \subset \mathcal{D}$ be the inclusion of a full subcategory. Given a functor $F: \mathcal{D} \rightarrow \mathcal{S}_{*}$, we can consider the fiber of the map of (homotopy) limits

$$
\lim _{\mathcal{D}} F \rightarrow \lim _{\mathcal{C}} F
$$

and ask when this is a functor of some iterated loop space of $F$ (rather than simply of $F$ ).

Definition 3. Let $\mathcal{C} \subset \mathcal{D}$ be as above. We say that the inclusion $\mathcal{C} \rightarrow \mathcal{D}$ is $k$-fold deloopable if there is an accessible, limit-preserving functor $\mathscr{G}: \operatorname{Fun}\left(\mathcal{D}, \mathcal{S}_{*}\right) \rightarrow \mathcal{S}_{*}$ and a natural equivalence

$$
\mathscr{G}\left(\Omega^{k} F\right) \simeq \operatorname{fib}\left(\lim _{\mathcal{D}} F \rightarrow \lim _{\mathcal{C}} F\right), \quad F \in \operatorname{Fun}\left(\mathcal{D}, \mathcal{S}_{*}\right) .
$$

In other words, the fiber of (2) should functorially factor through $\Omega^{k}$.

Example 4. By the discussion in the introduction, $\Delta^{\leq n-1} \subset \Delta^{\leq n}$ is $n$-fold deloopable. 
We begin by proving some elementary properties of $k$-fold deloopability.

Proposition 5. Let $\mathcal{C} \subset \mathcal{D}$ be a fully faithful inclusion. Then the following are equivalent:

(1) $\mathcal{C} \subset \mathcal{D}$ is $k$-fold deloopable.

(2) Let $\operatorname{Fun}_{\mathcal{C}}\left(\mathcal{D}, \mathcal{S}_{*}\right)$ be the full subcategory of $\operatorname{Fun}\left(\mathcal{D}, \mathcal{S}_{*}\right)$ consisting of those functors that take $\mathcal{C}$ to $*$. Then the functor

$$
\underbrace{\lim }_{\mathcal{D}}: \operatorname{Fun}_{\mathcal{C}}\left(\mathcal{D}, \mathcal{S}_{*}\right) \rightarrow \mathcal{S}_{*}
$$

factors through $\Omega^{k}$. In other words, there is an accessible, limit-preserving functor $\mathscr{H}: \operatorname{Fun}_{\mathcal{C}}\left(\mathcal{D}, \mathcal{S}_{*}\right) \rightarrow \mathcal{S}_{*}$ together with a natural identification $\varliminf_{\mathcal{D}} \simeq$ $\mathscr{H} \circ \Omega^{k}$ of functors $\operatorname{Fun}_{\mathcal{C}}\left(\mathcal{D}, \mathcal{S}_{*}\right) \rightarrow \mathcal{S}_{*}$.

Proof. Suppose $\mathcal{C} \subset \mathcal{D}$ is $k$-fold deloopable. Then, for $F \in \operatorname{Fun}_{\mathcal{C}}\left(\mathcal{D}, \mathcal{S}_{*}\right), \lim _{\mathcal{D}} F$ is naturally equivalent to the fiber of $\lim _{\mathcal{D}} F \rightarrow \lim _{\mathcal{C}} F$ since $\left.F\right|_{\mathcal{C}}$ is constant at $*$. But this factors through $\Omega^{k}$ by $k$-fold deloopability.

Conversely, suppose the second condition is satisfied. In this case, we note that for any $F \in \operatorname{Fun}\left(\mathcal{D}, \mathcal{S}_{*}\right)$, we have

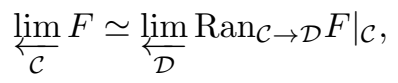

where $\operatorname{Ran}_{\mathcal{C} \rightarrow \mathcal{D}}$ denotes the right Kan extension from $\mathcal{C}$ to $\mathcal{D}$. We refer to [11, §4.3] for a treatment of Kan extensions for $\infty$-categories. In particular, the fiber of (2) can be identified with

$$
\underbrace{\lim _{\operatorname{fib}}}_{\mathcal{D}}\left(\left.F \rightarrow \operatorname{Ran}_{\mathcal{C} \rightarrow \mathcal{D}} F\right|_{\mathcal{C}}\right)
$$

and the functor $\operatorname{fib}\left(\left.F \rightarrow \operatorname{Ran}_{\mathcal{C} \rightarrow \mathcal{D}} F\right|_{\mathcal{C}}\right) \in \operatorname{Fun}\left(\mathcal{D}, \mathcal{S}_{*}\right)$ has the property that it takes the value $*$ on $\mathcal{C} \subset \mathcal{D}$, since $\mathcal{C} \subset \mathcal{D}$ is a full subcategory. Therefore, by the second condition, this is a functor of $\Omega^{k} F$.

Corollary 6. Let $i: \mathcal{C} \rightarrow \mathcal{D}$ be a fully faithful inclusion. Let $p: \mathcal{D}^{\prime} \rightarrow \mathcal{D}$ be a right cofinal functor and let $\mathcal{C}^{\prime} \subset \mathcal{D}^{\prime}$ be the subcategory of all objects that map, under $p$, into the essential image of $i$. Then if $\mathcal{C}^{\prime} \subset \mathcal{D}^{\prime}$ is $k$-fold deloopable, so is $\mathcal{C} \subset \mathcal{D}$.

Proof. To see this, we use the criterion of Proposition 5. Consider any functor $F: \mathcal{D} \rightarrow \mathcal{S}_{*}$ that maps everything in $\mathcal{C}$ to a weakly contractible space. Then $F \circ p$ maps everything in $\mathcal{C}^{\prime} \subset \mathcal{D}^{\prime}$ to $*$. In particular, $F \mapsto \varliminf_{\mathcal{D}} F=\varliminf_{\lim _{\mathcal{D}^{\prime}}} F \circ p$ is a functor of $\Omega^{k} F$.

We now give a basic criterion for $k$-fold deloopability. Consider the $\infty$-category $\operatorname{Fun}\left(\mathcal{D}, \mathcal{S}_{*}\right)$ of functors $\mathcal{D} \rightarrow \mathcal{S}_{*}$. If $F \in \operatorname{Fun}\left(\mathcal{D}, \mathcal{S}_{*}\right)$, then the homotopy $\operatorname{limit}_{\lim _{\mathcal{D}}} F$ is the (pointed) mapping space $\operatorname{Hom}_{\mathrm{Fun}\left(\mathcal{D}, \mathcal{S}_{*}\right)}\left(*_{+}, F\right)$, where $*_{+}$is the constant functor with value $*_{+}=S^{0}$. Analogously, the homotopy limit $\lim _{\mathcal{C}} F$ is given by

$$
\lim _{\mathcal{C}} F \simeq \operatorname{Hom}_{\mathrm{Fun}\left(\mathcal{C}, \mathcal{S}_{*}\right)}\left(*_{+},\left.F\right|_{\mathcal{C}}\right) .
$$

Now let $\operatorname{Lan}_{\mathcal{C} \rightarrow \mathcal{D}}$ denote the left Kan extension operation, from functors out of $\mathcal{C}$ to functors out of $\mathcal{D}$. It follows by adjointness that

$$
\lim _{\mathcal{C}} F \simeq \operatorname{Hom}_{F u n\left(\mathcal{D}, \mathcal{S}_{*}\right)}\left(\operatorname{Lan}_{\mathcal{C} \rightarrow \mathcal{D}}\left(*_{+}\right), F\right),
$$


and the natural forgetful map (2) is obtained from the counit map

$$
\operatorname{Lan}_{\mathcal{C} \rightarrow \mathcal{D}}\left(*_{+}\right) \rightarrow *_{+},
$$

in $\operatorname{Fun}\left(\mathcal{D}, \mathcal{S}_{*}\right)$. Therefore, it follows that the fiber of (2) (over the given basepoint, that is) is given by the functor

$$
F \mapsto \operatorname{Hom}_{\operatorname{Fun}\left(\mathcal{D}, \mathcal{S}_{*}\right)}(T, F),
$$

where the functor $T=T_{\mathcal{C}, \mathcal{D}}: \mathcal{D} \rightarrow \mathcal{S}_{*}$ is the homotopy pushout

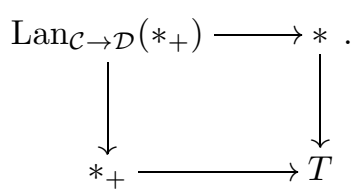

When the functor $T: \mathcal{D} \rightarrow \mathcal{S}_{*}$ can be desuspended $k$ times, so that $T \simeq \Sigma^{k} T^{\prime}$ for some $T^{\prime} \in \operatorname{Fun}\left(\mathcal{D}, \mathcal{S}_{*}\right)$, then the fiber of (2) is given by $\Omega^{k} \operatorname{Hom}_{\text {Fun }\left(\mathcal{D}, \mathcal{S}_{*}\right)}\left(T^{\prime}, F\right)$. The main strategy of this paper is to use a direct obstruction-theoretic argument to make such desuspensions.

Combining these ideas, we get:

Proposition 7. Let $\mathcal{C} \subset \mathcal{D}$ be an inclusion of a full subcategory. Then the following are equivalent:

(1) $\mathcal{C} \subset \mathcal{D}$ is $k$-fold deloopable.

(2) The functor $T: \mathcal{D} \rightarrow \mathcal{S}_{*}$ defined by (3) can be functorially desuspended $k$ times.

Proof. The fiber of (2), as a functor $\operatorname{Fun}\left(\mathcal{D}, \mathcal{S}_{*}\right) \rightarrow \mathcal{S}_{*}$, is corepresentable by $T$. Thus, by Yoneda's lemma, to deloop the functor (2) $k$ times by a corepresentable functor is equivalent to desuspending the corepresenting object $T k$ times. Any functor $\mathscr{G}$ as in Definition 3 is corepresentable by the adjoint functor theorem [11, Proposition 5.5.2.7].

There is no reason to restrict to functors taking values in $\mathcal{S}_{*}$ : we could take any pointed $\infty$-category with enough limits. For example, we could take $\mathcal{S}_{*}^{\text {op }}$ so as to get a result about $\mathcal{C}$-valued and $\mathcal{D}$-valued homotopy colimits of spaces. Indeed, let $\mathcal{A}$ be any pointed $\infty$-category with limits. In this case, given a functor $f: \mathcal{D} \rightarrow \mathcal{S}_{*}$ and a functor $F: \mathcal{D} \rightarrow \mathcal{A}$, we can form an internal mapping object $\operatorname{Hom}_{\mathrm{Fun}(\mathcal{D}, \mathcal{A})}(f, F) \in$ $\mathcal{A}$. This construction is determined by the properties that it should send homotopy colimits in $f$ to homotopy limits and that if $f$ is $\operatorname{Hom}(d, \cdot)_{+}$for $d \in \mathcal{D}$, then $\operatorname{Hom}_{\mathrm{Fun}(\mathcal{D}, \mathcal{A})}(f, F) \in \mathcal{A}$ is given by $F(d)$. If $f$ is $*_{+}$, then one gets the homotopy limit of $F$. In particular, we find that the fiber of the map $\lim _{\mathcal{D}} F \rightarrow \lim _{\mathcal{C}} F$ can be identified with $\operatorname{Hom}_{\operatorname{Fun}(\mathcal{D}, \mathcal{A})}(T, F)$ as before. In particular, if $T$ is $k$-fold desuspendable, then this fiber can be obtained as a $k$-fold loop space. We state this formally.

Corollary 8. Let $\mathcal{A}$ be any pointed $\infty$-category with limits. Then if $\mathcal{C} \subset \mathcal{D}$ is a $k$-fold deloopable inclusion, the functor $\operatorname{Fun}(\mathcal{D}, \mathcal{A}) \rightarrow \mathcal{A}$ sending $F \in \operatorname{Fun}(\mathcal{D}, \mathcal{A})$ to the fiber of $\varliminf_{\mathcal{D}} F \rightarrow \lim _{\mathcal{C}} F$ can be expressed as $\mathscr{G} \circ \Omega^{k}$ for a limit-preserving functor $\mathscr{G}: \operatorname{Fun}(\mathcal{D}, \mathcal{A}) \rightarrow \overleftarrow{\mathcal{A}}$ 
We recall, for future reference, the explicit construction of the left Kan extension, in the case where $\mathcal{C}, \mathcal{D}$ are ordinary categories. Given ordinary categories $\mathcal{C} \subset \mathcal{D}$, the left Kan extension $\operatorname{Lan}_{\mathcal{C} \rightarrow \mathcal{D}}(*): \mathcal{D} \rightarrow \mathcal{S}$ of the functor $\mathcal{C} \rightarrow \mathcal{S}$ constant at $*$ (where we work with unpointed spaces) given by the functor to the category of simplicial sets

$$
\mathcal{D} \rightarrow \mathcal{S}, \quad d \mapsto N\left(\mathcal{C}_{/ d}\right)
$$

where $\mathcal{C}_{/ d}$ is the fiber product $\mathcal{C} \times{ }_{\mathcal{D}} \mathcal{D} / d$ for $\mathcal{D}_{/ d}$ the overcategory. Note in particular that $T(c)$ is weakly contractible, for any $c \in \mathcal{C}$.

Remark 9. Observe that (3) exhibits $T$ as an unreduced suspension of the functor $\operatorname{Lan}_{\mathcal{C} \rightarrow \mathcal{D}}(*): \mathcal{D} \rightarrow \mathcal{S}$. It follows that if $\operatorname{Lan}_{\mathcal{C} \rightarrow \mathcal{D}}(d)$ is a nonempty space for each $d \in \mathcal{D}$, then each $T(d) \in \mathcal{S}_{*}$ can be written as a suspension in $\mathcal{S}_{*}$. In our primary example of interest, $T(d)$ is a wedge of spheres for each $d$. Thus, even the individual maps $T\left(d_{1}\right) \rightarrow T\left(d_{2}\right)$ will be suspension maps. As a result, the composite functor $\mathcal{D} \stackrel{T}{\rightarrow} \mathcal{S}_{*} \rightarrow \operatorname{Ho}\left(\mathcal{S}_{*}\right)$ factors through $\Sigma$. However, that alone does not mean we can desuspend the functor $T$.

The distinction between diagrams in the homotopy category and in the actual $\infty$-category $\mathcal{S}_{*}$ (e.g., presented by a model category such as that of simplicial sets) is central to this paper. These ideas are classical and have been studied in detail by Cooke [5] for group actions and more generally by Dwyer and Kan in [8 10]. We will not need the sophisticated cohomological machinery that these authors develop, though.

\section{Obstruction theORY}

Let $\mathcal{C} \subset \mathcal{C}^{\prime}$ be an inclusion. In order to understand whether the functor $T_{\mathcal{C}, \mathcal{C}^{\prime}}: \mathcal{C}^{\prime}$ $\rightarrow \mathcal{S}_{*}$ introduced in the previous section can be desuspended a certain number of times, we will need to introduce a small amount of obstruction theory for $\infty$ categories. While such obstruction theories are (in various forms) classical, we will use very little. It will be convenient to use the quasicategory model of $\infty$-categories.

Let $K$ be a simplicial set. Let $\mathcal{C}, \mathcal{D}$ be quasicategories and $F: \mathcal{C} \rightarrow \mathcal{D}$ a functor (not necessarily an inclusion). In our setting, it will be the suspension functor $\Sigma: \mathcal{S}_{*} \rightarrow \mathcal{S}_{*}$ restricted to a subcategory of $\mathcal{S}_{*}$, as we are trying to desuspend the functor $T$ from the previous section. Our goal is to contemplate lifting diagrams of the form

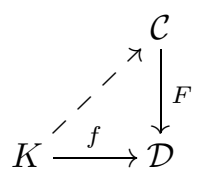

Such a lifting diagram is understood to take place in the homotopy category of quasicategories (e.g., of the Joyal model structure on simplicial sets).

Proposition 10. Suppose $F: \mathcal{C} \rightarrow \mathcal{D}$ has the property that the essential image of $F$ contains that of $f$. Suppose $\operatorname{dim} K \leq m$ and suppose that for each $c_{1}, c_{2} \in \mathcal{C}$, the map

$$
F: \operatorname{Hom}_{\mathcal{C}}\left(c_{1}, c_{2}\right) \rightarrow \operatorname{Hom}_{\mathcal{D}}\left(F c_{1}, F c_{2}\right)
$$

is $(m-1)$-connected. Then a lift in (4) always exists. 
Definition 11. For $m \geq 0$, we will say that a functor $F: \mathcal{C} \rightarrow \mathcal{D}$ is $m$-connected if $F$ is essentially surjective and for each $c_{1}, c_{2} \in \mathcal{C}$, the map $F: \operatorname{Hom}_{\mathcal{C}}\left(c_{1}, c_{2}\right) \rightarrow$ $\operatorname{Hom}_{\mathcal{D}}\left(F c_{1}, F c_{2}\right)$ is $(m-1)$-connected.

Proof. In view of the equivalence between quasicategories and simplicial categories [11, Theorem 2.2.5.1], we can assume without loss of generality that the quasicategories $\mathcal{C}, \mathcal{D}$ are the homotopy coherent nerves of fibrant simplicial categories $\mathscr{C}, \mathscr{D}$ (using the model structure of Bergner [2]). Moreover, we can assume that $F: \mathcal{C} \rightarrow \mathcal{D}$ is the homotopy coherent nerve of a simplicially enriched functor $F: \mathscr{C} \rightarrow \mathscr{D}$ which in turn is a fibration in the Bergner model structure. In this case, we claim that any lifting problem (4) can be solved in the category of simplicial sets itself. Inducting over the skeleta, it now suffices to show that in any diagram of simplicial sets, for $k \leq m$,

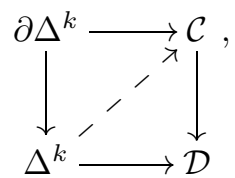

there exists a lift.

In this case, we need to solve the equivalent lifting problem (in simplicial categories) for

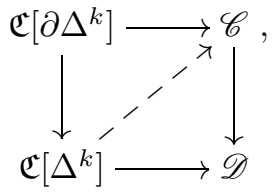

where we have used the notation as in 11, Def. 1.1.5.1]. The equivalence follows from the definition of the homotopy coherent nerve as the right adjoint to $\mathfrak{C}$.

However, we note that the simplicial functor $\mathfrak{C}\left[\partial \Delta^{k}\right] \rightarrow \mathfrak{C}\left[\Delta^{k}\right]$ is obtained via a pushout along a cofibration in the Bergner model structure. For a simplicial set $L$, let $\mathfrak{D}[L]$ denote the simplicial category with two objects $a, b$ with $\operatorname{Hom}(a, a)=$ $\operatorname{Hom}(b, b)=\Delta^{0}, \operatorname{Hom}(a, b)=L, \operatorname{Hom}(b, a)=\emptyset$. Then there is a pushout diagram in simplicial categories

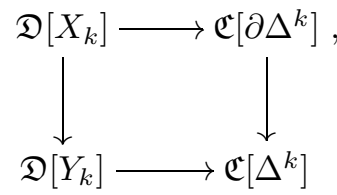

where $X_{k}$ has the homotopy type of $S^{k-2}$ and $Y_{k}$ the homotopy type of a point; moreover, $X_{k} \rightarrow Y_{k}$ is a cofibration. For this, compare the discussion on [11, p. 90] in the proof of [11, Theorem 2.2.5.1]. Therefore, our lifting problem is equivalent to that given by the diagram of simplicial categories

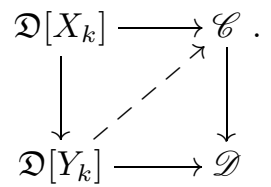


Unwinding the definitions, it follows that our lifting problem (5) is equivalent to a lifting problem in simplicial sets

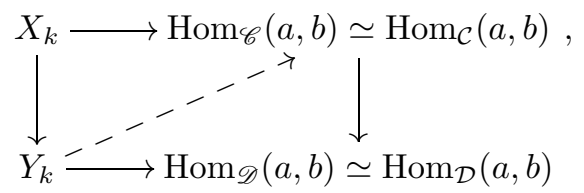

and we claim that we can solve this by the connectivity hypotheses.

In fact, suppose $U \rightarrow U^{\prime}$ is a fibration of Kan complexes which is $(s-1)$ connected. Suppose $r \leq s-1$. Then in any diagram of simplicial sets

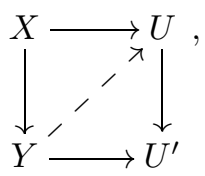

with $X \rightarrow Y$ a cofibration such that $X$ has the homotopy type of $S^{r-1}$ and $Y$ has the homotopy type of $*$, there exists a lift by obstruction theory. In our case, note that $\operatorname{Hom}_{\mathscr{C}}(a, b) \rightarrow \operatorname{Hom}_{\mathscr{D}}(a, b)$ is a Kan fibration because $\mathscr{C} \rightarrow \mathscr{D}$ is a fibration of simplicial categories.

Remark 12. The simplicial sets that one encounters in the above argument will not all be quasicategories, even if $K$ is a quasicategory. It is a convenient feature of the simplicial model that one can still make "cell" decompositions in this way.

In the rest of this section, we will note two simple tools for working with the above ideas which we will use in the sequel.

Proposition 13. Fix an integer $m$. Let $\mathcal{C}$ be an ordinary category with the property that whenever $c_{0}, \ldots, c_{m+1}$ are given objects in $\mathcal{C}$ and $c_{i} \rightarrow c_{i+1}$, for all $0 \leq i \leq m$, are given morphisms, then (at least) one of the morphisms is the identity. Then the nerve of $\mathcal{C}$ has dimension $\leq m$ as a simplicial set.

For instance, if $\mathcal{C}$ is a poset, then it follows that the dimension is one less than the maximum size of a chain.

Proof. In fact, every nondegenerate simplex in the nerve of $\mathcal{C}$ consists of such a tuple of morphisms as above with none of the identity, so that every nondegenerate simplex must have dimension $\leq m$.

Finally, we include an auxiliary result that enables us to simplify the process of finding liftings by leaving out certain parts of the category.

Proposition 14. Let $P$ be a poset and $P^{\prime} \subset P$ a subposet such that no element of $P^{\prime}$ is less than any element of $P \backslash P^{\prime}$. Let $\operatorname{Fun}^{\prime}\left(N(P), \mathcal{S}_{*}\right)$ denote the full subcategory of $\operatorname{Fun}\left(N(P), \mathcal{S}_{*}\right)$ spanned by those functors that send $P \backslash P^{\prime}$ to $*$. Then the restriction functor

$$
\operatorname{Fun}^{\prime}\left(N(P), \mathcal{S}_{*}\right) \rightarrow \operatorname{Fun}\left(N\left(P^{\prime}\right), \mathcal{S}_{*}\right)
$$

is an equivalence of $\infty$-categories. 
Proof. Observe that as a simplicial set, $N(P)$ is built from $N\left(P^{\prime}\right)$ by iteratively attaching $n$-simplices $\Delta^{n}$ along the boundary $\partial \Delta^{n}$, where the initial vertex is necessarily in $P \backslash P^{\prime}$. In other words, there exists a finite sequence of simplicial sets

$$
N\left(P^{\prime}\right)=K_{0} \rightarrow K_{1} \rightarrow \cdots \rightarrow K_{r}=N(P),
$$

where each map $K_{i} \rightarrow K_{i+1}$ fits into a pushout square

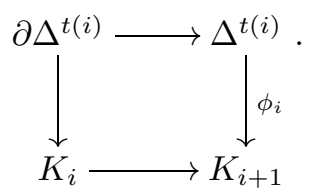

Moreover, the vertex 0 of each $\partial \Delta^{t(i)}$ is mapped to an element of $P \backslash P^{\prime}$. Note that $K_{i} \subset N(P)$, and we let $V_{i} \subset\left(K_{i}\right)_{0}$ denote the collection of vertices in $K_{i}$ that belong to $P \backslash P^{\prime}$. Note that $V_{i} \subset V_{i+1}$ with equality unless $t(i)=0$, in which case $V_{i+1}$ is the union of $V_{i}$ and the image of the map $\phi_{i}: \Delta^{0} \rightarrow K_{i+1}$.

For each $i$, we let $\operatorname{Fun}^{\prime}\left(K_{i}, \mathcal{S}_{*}\right)$ denote the subcategory of $\operatorname{Fun}\left(K_{i}, \mathcal{S}_{*}\right)$ spanned by those functors which carry every vertex in $V_{i}$ to $*$. We then have $\operatorname{Fun}^{\prime}\left(K_{0}, \mathcal{S}_{*}\right)=$ $\operatorname{Fun}\left(N\left(P^{\prime}\right), \mathcal{S}_{*}\right)$ and $\operatorname{Fun}^{\prime}\left(K_{r}, \mathcal{S}_{*}\right)=\operatorname{Fun}^{\prime}\left(N(P), \mathcal{S}_{*}\right)$. For each $i$, we have a pullback diagram of $\infty$-categories

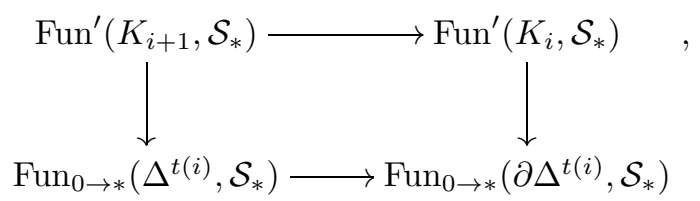

where the $0 \rightarrow *$ subscript indicates that the initial vertex is sent to $*$.

By induction, it thus suffices to show that the forgetful functor

$$
\operatorname{Fun}_{0 \rightarrow *}\left(\Delta^{n}, \mathcal{S}_{*}\right) \rightarrow \operatorname{Fun}_{0 \rightarrow *}\left(\partial \Delta^{n}, \mathcal{S}_{*}\right)
$$

is an equivalence of $\infty$-categories. This follows from the next lemma.

Lemma 15. Let $\mathcal{C}$ be a quasicategory containing an initial object and let $\operatorname{Fun}_{0 \rightarrow *}\left(\Delta^{n}, \mathcal{C}\right)$ be the full subcategory of the quasicategory $\operatorname{Fun}\left(\Delta^{n}, \mathcal{C}\right)$ spanned by those functors that send $0 \in \Delta^{n}$ to an initial object. Define $\operatorname{Fun}_{0 \rightarrow *}\left(\partial \Delta^{n}, \mathcal{C}\right)$ similarly. Then the restriction map

$$
\operatorname{Fun}_{0 \rightarrow *}\left(\Delta^{n}, \mathcal{C}\right) \rightarrow \operatorname{Fun}_{0 \rightarrow *}\left(\partial \Delta^{n}, \mathcal{C}\right)
$$

is a trivial fibration of simplicial sets.

Proof. For $n=0$, this assertion states that the subcategory of $\mathcal{C}$ spanned by the initial objects is a contractible Kan complex, which is proved in 11, Proposition 1.2.12.9]. We thus assume $n \geq 1$. We need to show that any lifting problem

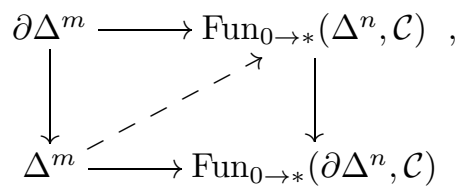


can be solved. This lifting problem is equivalent to one of the form

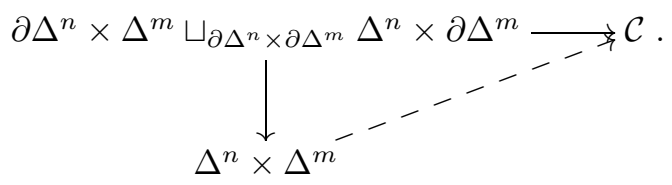

The given map $\partial \Delta^{n} \times \Delta^{m} \sqcup_{\partial \Delta^{n} \times \partial \Delta^{m}} \Delta^{n} \times \partial \Delta^{m} \rightarrow \mathcal{C}$ carries every vertex $(0, i)$ (for $i \in\{0,1, \ldots, m\})$ to an initial object of $\mathcal{C}$. Moreover, the inclusion of simplicial sets $\partial \Delta^{n} \times \Delta^{m} \sqcup_{\partial \Delta^{n} \times \partial \Delta^{m}} \Delta^{n} \times \partial \Delta^{m} \rightarrow \Delta^{n} \times \Delta^{m}$ is built up by successively attaching simplices $\Delta^{p}$, along the boundary $\partial \Delta^{p}$, whose initial vertex is $(0,0)$. In particular, to find the lift in (6) , it suffices to contemplate a problem of the form

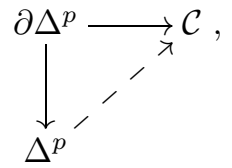

where the initial vertex of $\partial \Delta^{p}$ maps to an initial object of $\mathcal{C}$. But by the definition of an initial object of a quasicategory [11, Definition 1.2.12.3], such lifting problems can always be solved.

\section{Specialization to totalizations}

In this section, we will prove our main result on $k$-fold deloopability for totalizations (which is a restatement of Theorem 1).

Theorem 16. Let $1 \leq n \leq m \leq 2 n+1$. Then $\Delta^{\leq n} \subset \Delta^{\leq m}$ is $(2 n-m+2)$-fold deloopable.

In order to prove Theorem 16, we will follow the outline already laid out in the previous sections. Namely, we will write down the functor $T$ introduced in Section 2 explicitly; it will be pointwise a wedge of spheres. Then we will show, using the obstruction theory, that it can be desuspended the right number of times.

Our first problem is, however, that the nerve $N\left(\Delta^{\leq n}\right)$ is not a finite-dimensional simplicial set, and it will be convenient to replace it by one.

Definition 17. As before, let $\Delta$ be the simplex category, of finite nonempty totally ordered sets and order-preserving maps between them. We let $\Delta^{\text {inj }} \subset \Delta$ be the subcategory with the same objects but injective order-preserving maps. We let $\Delta^{\mathrm{inj}, \leq n}$ and $\Delta^{\leq n}$ denote the analogs where we only consider totally ordered sets of cardinality $\leq n+1$.

It is classical that the inclusion $\Delta^{\text {inj }} \subset \Delta$ is (homotopy) right cofinal; see for instance [15, Example 8.5.12]. The advantage of the category $\Delta^{\text {inj }}$ is that the nerve of a skeleton of $\Delta^{\mathrm{inj}, \leq n}$ is $n$-dimensional, as any composable sequence $x_{0} \rightarrow x_{1} \rightarrow$ $\cdots \rightarrow x_{n+1}$ of arrows in a skeleton of $\Delta^{\mathrm{inj}, \leq n}$ contains an identity. (Compare Proposition 13.) However, the inclusion $\Delta^{\mathrm{inj}, \leq n} \subset \Delta^{\leq n}$ is no longer homotopy final: $\Delta^{\leq n}$ is weakly contractible (as it has a terminal object) while $\Delta^{\text {inj, } \leq n}$ fails to be weakly contractible (for instance for $n=1$ ). Recall that any right cofinal map of quasicategories is in particular a weak homotopy equivalence of underlying simplicial sets [11, Proposition 4.1.1.3]. 
Let $\Delta_{/[n]}^{\mathrm{inj}, \leq n}$ be the overcategory; it is equivalent to the partially ordered set of all nonempty subsets of $[n]=\{0,1, \ldots, n\}$. We have a natural functor $\Delta_{/[n]}^{\mathrm{inj}, \leq n} \rightarrow$ $\Delta^{\mathrm{inj}, \leq n} \rightarrow \Delta \leq n$. Now we use:

Proposition 18. The composite $\Delta_{/[n]}^{\mathrm{inj}, \leq n} \rightarrow \Delta^{\leq n}$ is right cofinal.

References for Proposition 18, which is folklore but technical, include [6. Proposition 18.7] and [12, Lemma 1.2.4.17].

We will thus work with the $\Delta_{/[n]}^{\mathrm{inj}, \leq n}$. Using Corollary 6, it suffices to prove an analog of $k$-fold deloopability here; the advantage is that, by Proposition 13 , the nerve has dimension $n$ (up to taking a skeleton).

First, we will change notation. Let $S$ be a finite, nonempty set (e.g., $[n])$. We let $\mathcal{P}(S)$ denote the partially ordered set of all nonempty subsets of $S$, which by abuse of notation we will identify with its nerve. Let $\mathcal{P}_{\leq r}(S) \subset \mathcal{P}(S)$ be the partially ordered subset of all nonempty subsets of $S$ of cardinality $\leq r$. It follows by Corollary 6 and Proposition 18 that our main result, Theorem 16, is a corollary (with $r=n+1, S=[m],|S|=m+1$ ) of:

Proposition 19. $\mathcal{P}_{\leq r}(S) \subset \mathcal{P}(S)$ is $(2 r-|S|+1)$-fold deloopable.

Proof. The left Kan extension $\operatorname{Lan}_{\mathcal{P}_{\leq r}(S) \rightarrow \mathcal{P}(S)}(*)$ can be described explicitly by the following formula: it assigns to an arbitrary nonempty subset $U \subset S$ the nerve of the partially ordered set $\mathcal{P}_{\leq r}(U)$ of all nonempty subsets of $U$ of cardinality $\leq r$. In general, for any finite set $U$, the nerve of $\mathcal{P}_{\leq r}(U)$ is isomorphic to the barycentric subdivision of the simplicial complex obtained as the union of all the $(r-1)$-simplices of a $(|U|-1)$-simplex. This has the homotopy type of a wedge of $(r-1)$-spheres, as one sees by comparing the simplicial homology to that of the standard simplex. It follows that the functor

$$
T=T_{\mathcal{P}_{\leq r}(S) \rightarrow \mathcal{P}(S)}: \mathcal{P}(S) \rightarrow \mathcal{S}_{*}
$$

is, pointwise, a wedge of $r$-spheres: it is the unreduced suspension of the left Kan extension $\operatorname{Lan}_{\mathcal{P}_{\leq r}(S) \rightarrow \mathcal{P}(S)}(*)$. Moreover, the functor $T$ is weakly contractible when evaluated on any subset of $S$ of cardinality $\leq r$.

Our goal is to functorially desuspend $T$. Consider the subset $\mathcal{P}_{>r}(S)$ of the poset $\mathcal{P}(S)$ where we consider subsets of $S$ of cardinality $>r$. By Proposition 14, it is equivalent to desuspend $\left.T\right|_{\mathcal{P}_{>r}(S)}$. By Proposition 13, the nerve of $\mathcal{P}_{>r}(S)$ has dimension $|S|-r-1$.

Let $d>0$. We are thus contemplating the lifting problem

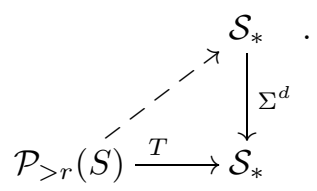

We do not need to work with all of $\mathcal{S}_{*}$ for this. For each $p$, consider the $\infty$ category $\mathcal{C}(p) \subset \mathcal{S}_{*}$ which is the full subcategory spanned by the finite wedges of $p$-spheres. One has a functor

$$
\Sigma: \mathcal{C}(p) \rightarrow \mathcal{C}(p+1)
$$


and our problem is to find a lift

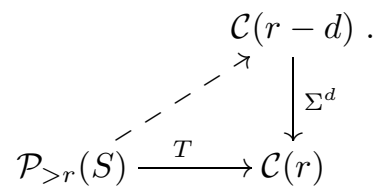

Observe that, for any $p$, the maps given by $\Sigma$,

$\operatorname{Hom}_{*}\left(S^{p} \vee \cdots \vee S^{p}, S^{p} \vee \cdots \vee S^{p}\right) \rightarrow \operatorname{Hom}_{*}\left(S^{p+1} \vee \cdots \vee S^{p+1}, S^{p+1} \vee \cdots \vee S^{p+1}\right)$, are $(p-1)$-connected by the Freudenthal suspension theorem. Therefore, the functor $\Sigma^{d}: \mathcal{C}(r-d) \rightarrow \mathcal{C}(r)$ above is $(r-d)$-connected in the sense of Definition 11. By Proposition 10, we can find a lift if

$$
\operatorname{dim} N\left(\mathcal{P}_{>r}(S)\right)=|S|-r-1 \leq r-d,
$$

or equivalently if

$$
d \leq 2 r-|S|+1,
$$

as desired. In particular, we can desuspend $T$ by $2 r-|S|+1$ times, which proves that the inclusion in question is $(2 r-|S|+1)$-fold deloopable.

Remark 20. By Theorem 16, we find that if $X_{\bullet}$ is any pointed simplicial space, then the cofiber of the geometric realization of the $m$-truncation by the geometric realization of the $n$-truncation is a $k$-fold suspension, for $k=2 n-m+2$. This cannot be improved, since (using the standard simplicial model given by the bar construction) $\mathbb{R} P^{m} / \mathbb{R P}^{n}$ cannot be a suspension if $m \geq 2 n+2$ and nontrivial cup products exist. In particular, Theorem 16 is best possible (at least for one-fold deloopings).

It will actually be convenient to have a slightly stronger result.

Corollary 21. Let $1 \leq n \leq m \leq 2 n+1$, let $k=2 n-m+2$ and let $\mathcal{C}$ be any pointed $\infty$-category with limits. Then for $X^{\bullet} \in \operatorname{Fun}(\Delta, \mathcal{C})$, the sequence of objects

$$
\operatorname{fib}\left(\operatorname{Tot}_{m}\left(X^{\bullet}\right) \rightarrow \operatorname{Tot}_{n}\left(X^{\bullet}\right)\right) \rightarrow \operatorname{fib}\left(\operatorname{Tot}_{m-1}\left(X^{\bullet}\right) \rightarrow \operatorname{Tot}_{n}\left(X^{\bullet}\right)\right) \rightarrow \cdots \rightarrow *
$$

is a functor of $\Omega^{k} X^{\bullet}$.

Each of the objects in question is naturally a functor of $\Omega^{k} X^{\bullet}$ by Theorem 16; the additional claim here is that the maps can be made into $k$-fold loop maps.

Proof. We consider the sequence of objects in $\operatorname{Fun}(\Delta, \mathcal{C})$,

$$
\operatorname{Ran}_{\Delta \leq m \rightarrow \Delta}\left(X^{\bullet}\right) \rightarrow \operatorname{Ran}_{\Delta \leq m-1 \rightarrow \Delta}\left(X^{\bullet}\right) \cdots \rightarrow \operatorname{Ran}_{\Delta \leq n \rightarrow \Delta}\left(X^{\bullet}\right) .
$$

Form the fibers of all of these over the last one to get a sequence of pointed cosimplicial spaces

$$
\left\{\operatorname{fib}\left(\operatorname{Ran}_{\Delta \leq i \rightarrow \Delta}\left(X^{\bullet}\right) \rightarrow \operatorname{Ran}_{\Delta \leq n \rightarrow \Delta}\left(X^{\bullet}\right)\right)\right\}_{n \leq i \leq m} .
$$

This is a sequence of pointed cosimplicial spaces, each of which restricts to $*$ on $\Delta \leq n$. When we take $\operatorname{Tot}_{m}$ of this sequence, the result is therefore a functor of $\Omega^{k} X^{\bullet}$ by Proposition 5 and Theorem 16. But when we take $\operatorname{Tot}_{m}$ of this sequence, we obtain precisely the sequence of pointed spaces in the statement of the corollary. 


\section{Applications And EXtensions}

5.1. Open covers. We start with an application to the most classical example of a homotopy colimit, which was stated in the introduction (Theorem 2).

Theorem 22. Let $X$ be a pointed topological space covered by $n$ open sets $U_{1}$, $U_{2}, \ldots, U_{n} \subset X$, each containing the basepoint. Suppose the intersection of any collection of at most $r$ of the $\left\{U_{i}\right\}$ is weakly contractible. Then $X$ has the weak homotopy type of a $(2 r-n+1)$-fold suspension.

Proof. For a space $X$ covered by open sets $U_{1}, \ldots, U_{n}$ as above, there is a weak equivalence [7] between $X$ and the homotopy colimit over the poset $\mathcal{P}(\{1,2, \ldots, n\})$ of the functor that sends a nonempty subset $S \subset\{1,2, \ldots, n\}$ to $\bigcap_{i \in S} U_{i}$. This is a contravariant functor from the poset to $\mathcal{S}_{*}$, and by assumption, it takes weakly contractible values on the subsets of $\{1,2, \ldots, n\}$ of cardinality $\leq r$. Using Proposition 19, we conclude that the homotopy colimit has the weak homotopy type of a $(2 r-n+1)$-fold suspension.

Suppose we are in the unpointed setting, and we have an open cover $U_{1}, \ldots, U_{n}$ of a space $X$ such that all the intersections of the $\left\{U_{i}\right\}$ are either empty or weakly contractible. In this case, $X$ has the weak homotopy type of the nerve of the cover, which has dimension $\leq n-1$ as a simplicial set. If the dimension is $n-1$, then $U_{1} \cap \cdots \cap U_{n} \neq \emptyset$ and is thus weakly contractible, so that $X$ is itself weakly contractible. Otherwise, the dimension is $\leq n-2$. We consider only this case.

Suppose the intersection of any $r$ of the $\left\{U_{i}\right\}$ is nonempty. Then, $X$ is $(r-2)$ connected. But any complex with cells in dimensions $[s, t]$ can be desuspended (by the Freudenthal suspension theorem) if $t \leq 2 s-1$. Here, we find that if $n-2 \leq 2(r-1)-1$, or $n \leq 2 r-1$, then, up to weak equivalence, $X$ can be desuspended (after choosing a basepoint). This is a version of the conclusion of Theorem 22 for a one-fold desuspension in an unpointed setting.

5.2. Other posets. In Proposition 19, we showed that if $\mathcal{P}(S)$ is the poset of nonempty subsets of a given finite set $S$ and $\mathcal{P}_{\leq r}(S) \subset \mathcal{P}(S)$ is the poset of nonempty subsets of cardinality $\leq r$, then the inclusion $\mathcal{P}_{\leq r}(S) \subset \mathcal{P}(S)$ is $(2 r-|S|+$ 1)-fold deloopable. We note here that other similar examples of deloopable poset inclusions occur "in nature". The study of homotopy types of posets and homotopy colimits of diagrams of spaces indexed over posets has numerous applications; see for instance [3, 14.

Let $k$ be a field, and let $V$ be an $n$-dimensional vector space over $k$. Consider the poset $\operatorname{Sub}(V)$ of nonzero subspaces of $V$. Given $2 \leq r \leq n$, let $\operatorname{Sub}_{\leq r}(V)$ be the poset of nonzero subspaces of $V$ which have dimension $\leq r$.

Proposition 23. The inclusion of posets $\operatorname{Sub}_{\leq r}(V) \subset \operatorname{Sub}(V)$ is $(2 r-n+1)$-fold deloopable.

Proof. Let $W \subset V$ be any subspace. We show that the nerve of $\operatorname{Sub}_{\leq r}(W)$ (i.e., the left Kan extension of a point from $\operatorname{Sub}_{\leq r}(V)$, evaluated on $W$ ) has the homotopy type of a wedge of $(r-1)$-spheres, following an argument of Folkman presented in [14, Proposition 8.6]: for each one-dimensional space $L \subset W$, we have a subposet $\mathrm{Sub}_{\leq r, L}(W)$ consisting of those $\leq r$-dimensional subspaces of $W$ that contain $L$. The intersection of any $r$ of the subposets $\operatorname{Sub}_{\leq r, L}(W)$ contains an initial object (i.e., minimal element) and is thus weakly contractible. Therefore, $\operatorname{Sub}_{\leq r}(W)$ is 
$(r-2)$-connected. However, the nerve of $\operatorname{Sub}_{\leq r}(W)$ has dimension $\leq r-1$ by Proposition 13. Putting these together, it follows that the nerve of $\mathrm{Sub}_{\leq r}(W)$ has the homotopy type of a wedge of $(r-1)$-spheres.

Now it follows that $T_{\mathrm{Sub}_{r}(V) \rightarrow \operatorname{Sub}(V)}$ is pointwise a wedge of $r$-spheres, and it is weakly contractible when evaluated on any subspace of dimension $\leq r$. Since the poset $\operatorname{Sub}(V) \backslash \operatorname{Sub}_{r}(V)$ has dimension $n-r-1$, the same argument as in Proposition 19 goes through.

5.3. Differentials. Let $X^{\bullet}$ be a pointed cosimplicial space, and consider the homotopy spectral sequence

$$
E_{2}^{s, t} \Longrightarrow \pi_{t-s} \operatorname{Tot} X^{\bullet},
$$

which comes from the Tot tower, and the differentials $d_{r}: E_{r}^{s, t} \rightarrow E_{r}^{s+r, t+r-1}$.

If $t-s>0$, the entire spectral sequence is a shift of the spectral sequence for $\Omega X^{\bullet}$. All the differentials mapping out of $E_{r}^{s, t}$ are determined by $\Omega X^{\bullet}$ if $t-s>0$. But there are also differentials $d_{r}: E_{r}^{s, s} \rightarrow E_{r}^{s+r, s+r-1}$ which measure the obstructions to finding points in $\pi_{0} \operatorname{Tot} X^{\bullet}$. These cannot be determined by $\Omega X^{\bullet}$, as they contribute to $\pi_{0}$. Nonetheless, the $E_{2}^{s, s}$ terms for $s>0$ are determined by $\Omega X^{\bullet}$.

Theorem 24. Given any cosimplicial space $X^{\bullet}$, any differential

$$
d_{r}: E_{r}^{s, s} \rightarrow E_{r}^{s+r, s+r-1}
$$

depends only on the cosimplicial space $\Omega X^{\bullet}$ for $r \leq s-1$.

Proof. The differentials $d_{k}, k \leq r$ starting in filtration $s$ are precisely the obstructions to lifting an element in $\operatorname{fib}\left(\operatorname{Tot}_{s}\left(X^{\bullet}\right) \rightarrow \operatorname{Tot}_{s-1}\left(X^{\bullet}\right)\right)$ to $\operatorname{fib}\left(\operatorname{Tot}_{r+s}\left(X^{\bullet}\right) \rightarrow\right.$ $\left.\operatorname{Tot}_{s-1}\left(X^{\bullet}\right)\right)$. In particular, if

$$
2(s-1)-(r+s)+2 \geq 1,
$$

then, by Corollary 21, this obstruction problem is determined entirely by $\Omega X^{\bullet}$.

Corollary 25. Suppose $X^{\bullet}$ is a pointed cosimplicial space with the properties:

(1) Any element in $\pi_{0} \operatorname{Tot} X^{\bullet}$ has filtration $\leq N$.

(2) Any class in $E_{2}^{s, s}$ for $s>N$ is either killed at a finite stage or supports a differential $d_{r}$ for $r \leq s-1$.

Then if $Y^{\bullet}$ is a path-connected cosimplicial space such that there exists an equivalence of pointed cosimplicial spaces $\Omega X^{\bullet} \simeq \Omega Y^{\bullet}$, the same conditions above are valid for the totalization of $Y^{\bullet}$.

Proof. This follows from the above theorem. Concretely, any element of the fiber fib $\left(\operatorname{Tot}_{s}\left(X^{\bullet}\right) \rightarrow \operatorname{Tot}_{s-1}\left(X^{\bullet}\right)\right)$ either maps to the connected component at $*$ in fib $\left(\operatorname{Tot}_{s}\left(X^{\bullet}\right) \rightarrow \operatorname{Tot}_{s-t-1}\left(X^{\bullet}\right)\right)$ for some $t>0$, which is detected by a differential that we see from the spectral sequence of $\Omega X^{\bullet}$ (i.e., not a "fringed" differential in the spectral sequence for $\left.X^{\bullet}\right)$, or it supports a "fringed" differential and fails to lift to $\operatorname{fib}\left(\operatorname{Tot}_{s+r}\left(X^{\bullet}\right) \rightarrow \operatorname{Tot}_{s-1}\left(X^{\bullet}\right)\right)$, where $r \leq s-1$. But in this range, everything only depends on $\Omega X^{\bullet}$ by Corollary 21 .

A natural example of the type of situation in Corollary 25 arises from a descendable map of $\mathbf{E}_{\infty}$-ring spectra $S \rightarrow R$. Denote by $R \bullet$ the cosimplicial $\mathbf{E}_{\infty}$-ring spectrum which is the cobar construction on the map $S \rightarrow R$, also known as the Amitsur complex. Descendability implies that the spectrum $S$ is equivalent to the totalization of $R^{\bullet}$. Under mild conditions, the spaces $B\left(\Omega^{\infty} R^{\bullet}\right)$ and $B G L_{1}\left(R^{\bullet}\right)$ 
satisfy the hypotheses of Corollary 25, since for an $\mathbf{E}_{1}$-ring spectrum $R$, the space $G L_{1}(R) \simeq \Omega B G L_{1}(R)$ is a union of connected components of $\Omega B\left(\Omega^{\infty} R\right)$. We discuss this example further in [13]: it was the motivating example and application for us.

\section{ACKNOWLEDGMENTS}

This result started out as a piece of the work in 13 on the calculation of Picard groups of certain ring spectra. We are grateful to Mike Hopkins for suggesting the original project and for several discussions, and to Haynes Miller for helpful comments.

\section{REFERENCES}

[1] Gregory Z. Arone, William G. Dwyer, and Kathryn Lesh, Loop structures in Taylor towers, Algebr. Geom. Topol. 8 (2008), no. 1, 173-210, DOI 10.2140/agt.2008.8.173. MR2377281 (2008m:55025)

[2] Julia E. Bergner, A model category structure on the category of simplicial categories, Trans. Amer. Math. Soc. 359 (2007), no. 5, 2043-2058, DOI 10.1090/S0002-9947-06-03987-0. MR2276611(2007i:18014)

[3] Anders Björner, Michelle L. Wachs, and Volkmar Welker, Poset fiber theorems, Trans. Amer. Math. Soc. 357 (2005), no. 5, 1877-1899, DOI 10.1090/S0002-9947-04-03496-8. MR2115080 (2006a:05172)

[4] A. K. Bousfield and D. M. Kan, Homotopy limits, completions and localizations, Lecture Notes in Mathematics, Vol. 304, Springer-Verlag, Berlin-New York, 1972. MR0365573 (51 \#1825)

[5] George Cooke, Replacing homotopy actions by topological actions, Trans. Amer. Math. Soc. 237 (1978), 391-406. MR0461544 (57 \#1529)

[6] Daniel Dugger, A primer on homotopy colimits, Preprint available at http://math.uoregon. edu/ ddugger/hocolim.pdf.

[7] Daniel Dugger and Daniel C. Isaksen, Topological hypercovers and $\mathbb{A}^{1}$-realizations, Math. Z. 246 (2004), no. 4, 667-689, DOI 10.1007/s00209-003-0607-y. MR2045835(2005d:55026)

[8] W. G. Dwyer and D. M. Kan, An obstruction theory for diagrams of simplicial sets, Nederl. Akad. Wetensch. Indag. Math. 46 (1984), no. 2, 139-146. MR749527 (86c:55010c)

[9] W. G. Dwyer and D. M. Kan, Realizing diagrams in the homotopy category by means of diagrams of simplicial sets, Proc. Amer. Math. Soc. 91 (1984), no. 3, 456-460, DOI 10.2307/2045321. MR744648 (86c:55010b)

[10] W. G. Dwyer, D. M. Kan, and J. H. Smith, An obstruction theory for simplicial categories, Nederl. Akad. Wetensch. Indag. Math. 48 (1986), no. 2, 153-161. MR849715 (87m:55023)

[11] Jacob Lurie, Higher topos theory, Annals of Mathematics Studies, vol. 170, Princeton University Press, Princeton, NJ, 2009. MR2522659 (2010j:18001)

[12] Jacob Lurie, Higher algebra, Preprint available at http://www.math.harvard.edu/ lurie, 2012.

[13] Akhil Mathew and Vesna Stojanoska, The Picard group of topological modular forms via descent theory, Preprint available at http://arxiv.org/abs/1409.7702, 2014.

[14] Daniel Quillen, Homotopy properties of the poset of nontrivial p-subgroups of a group, Adv. in Math. 28 (1978), no. 2, 101-128, DOI 10.1016/0001-8708(78)90058-0. MR493916 (80k:20049)

[15] Emily Riehl, Categorical homotopy theory, New Mathematical Monographs, vol. 24, Cambridge University Press, Cambridge, 2014. MR3221774

Department of Mathematics, University of California, Berkeley, California, 94720 E-mail address: amathew@math.berkeley.edu

Max Planck Institute for Mathematics, Bonn, Germany, 53111

E-mail address: vstojanoska@mpim-bonn.mpg.de 